\title{
ASPECTOS REPRODUCTI VOS DEL PEJERREY PATAGÓNICO (Odontesthes hatcheri Eigenmann, 1909) EN EL EMBALSE EZEQUI EL RAMOS MEXIA (ARGENTINA)
}

\section{REPRODUCTI VE ASPECTS OF THE PATAGONI AN SI LVERSIDE (Odontesthes hatcheri Eigenmann, 1909) FROM THE EZEQUIEL RAMOS MEXI A RESERVOI R (ARGENTI NA)}

\author{
Alejandro Dománico A, ${ }^{1 *} \mathrm{Ph} . \mathrm{D}$, Lauce Freyre $\mathrm{R},{ }^{2}$ Lic. \\ ${ }^{1}$ Comisión de Investigaciones Científicas de la Provincia de Buenos Aires ( $\mathrm{ClC}$ ). ${ }^{2}$ Consejo \\ Nacional de Investigaciones Científicas y Técnicas (CONICET). ${ }^{1,2}$ Instituto de Limnología \\ “Dr. Raúl A. Ringuelet" (FCNyM - CONICET) Avda. Calchaquí Km. 23.5 (1888) Florenci Varela, \\ Buenos Aires, Argentina. ${ }^{*}$ Correspondencia: aledoma_4_8@yahoo.com.ar
}

Recibido: Julio 2 de 2008; Aceptado: Diciembre 10 de 2008

\section{RESUMEN}

Objetivo. Estudiar el ciclo sexual y la fecundidad del pejerrey patagónico, especie de alto valor comercial y deportivo, en el embalse Ezequiel Ramos Mexía (Neuquén, Río Negro; Argentina). Materiales y métodos. Se colectaron 9307 ejemplares. El ciclo sexual se estudió mediante la observación macroscópica de las gónadas y la fecundidad absoluta se calculó con la técnica volumétrica. Resultados. La talla de primera madurez sexual $\left(\mathrm{L}_{\mathrm{m}} 50 \%\right)$ se estimó a través del ajuste de una curva logística. El desove tuvo lugar en primavera y principios de verano (septiembre a diciembre). La talla de primera madurez sexual, en las hembras, fue de $244,37 \mathrm{~mm}\left(\mathrm{~L}_{\mathrm{t}}\right)$. La fecundidad absoluta varió entre 3.976 ovocitos en una hembra de $296 \mathrm{~mm}\left(\mathrm{~L}_{\mathrm{t}}\right)$ y $190 \mathrm{~g}$ y 20.879 ovocitos en una hembra de $420 \mathrm{~mm}\left(\mathrm{~L}_{\mathrm{t}}\right)$ y $704 \mathrm{~g}$. Conclusiones. No se observó una relación significativa entre la fecundidad relativa y la longitud total de los ejemplares. La proporción de sexos resultó equilibrada en las clases de longitud intermedias, pero mostró una preponderancia de hembras en las mayores. El ciclo ovárico es altamente estacional y coincide con el de poblaciones de la especie en otros ambientes patagónicos. La fecundidad, en cambio, resultó más elevada que la observada en el lago Moreno, situado en la región cordillerana.

Palabras clave: Odontesthes hatcheri, fecundidad, ciclo sexual, pejerrey patagónico. 


\section{ABSTRACT}

Objective. To study the sexual cycle and fecundity of the Patagonian silverside, a species of high commercial and sport value, from Ezequiel Ramos Mexia reservoir (Neuquen, Rio Negro; Argentina). Materials and methods. Sampling was conducted between 1982 and 1986, and 9307 specimens were collected. The sexual cycle was studied by macroscopic observation of the gonads and fecundity was assessed using the volumetric method. Results. Length at first maturity $\left(\mathrm{L}_{\mathrm{m}} 50 \%\right)$ was estimated by fitting a logistic curve. Spawning took place in spring and early summer (September to December). Length at first maturity was $244.37 \mathrm{~mm}$ (Lt) in females. Absolute fecundity ranged between 3.976 oocytes in a $296 \mathrm{~mm}$ (Lt), $190 \mathrm{~g}$ female and 20.879 oocytes in a $420 \mathrm{~mm}$ (Lt), $704 \mathrm{~g}$ female. Conclusions. No significant relation was found between relative fecundity and total length. The sex ratio was approximately 1:1 at intermediate length classes, but females predominated in the larger classes. The ovarian cycle was highly seasonal and coincided with that of populations from other Patagonian environments. Fecundity, on the other hand, was higher than that observed at Moreno Lake, in the Andean region.

Key words: Odontesthes hatcheri, fecundity, sexual cycle, patagonian silverside.

\section{NTRODUCCIÓN}

La reproducción es uno de los fenómenos más relevantes dentro del ciclo biológico de todo ser viviente. Los biólogos pesqueros han prestado mucha atención a este parámetro, sobre todo cuando se trata de un recurso que es factible de explotación. El ciclo sexual y la fecundidad de peces son parámetros importantes desde el punto de vista de la dinámica de poblaciones, así como lo es conocer la tasa de natalidad de una población, que es el número de nuevos individuos producidos por unidad de tiempo.

Dentro de los aterinomorfos el pejerrey bonaerense es la especie que posee una mayor información sobre aspectos reproductivos, en cambio la información existente en el pejerrey patagónico es muy escasa. Existen algunos trabajos sobre el pejerrey patagónico:

Viozzi (1) estimó el índice gonadosomático (IGS) para un período anual, en muestras provenientes del lago Pellegrini (Pcia. del Neuquén). Bello et al (2) estudiaron, el índice gonadosomático en el pejerrey patagónico de la laguna Ñe Luán (Río Negro) Bello et al (3), realizaron estudios macroscópicos e histológicos de las gónadas de individuos de esta especie, provenientes del lago Moreno (Río Negro). Un estudio realizado por Ruiz (4), analizó los aspectos reproductivos de
Odontesthes hatcheri en muestras colectadas en el embalse Florentino Ameghino (Chubut).

Para conocer la época de reproducción se debe realizar un seguimiento del estado de madurez de las gónadas, al menos durante un período anual. La maduración gonadal, tanto en ovarios como en testículos comprende una serie de cambios los cuales pueden ser: variación de tamaño y forma, color, posición en la cavidad del cuerpo, entre otros, que obviamente conllevan a la producción de gametos tanto femeninos como masculinos.

Cuando se realizan estudios de producción o dinámica poblacional, es de suma importancia calcular la fecundidad específica por edad. Este valor depende de la fecundidad total o absoluta, de la talla de la primera madurez sexual y de la proporción de hembras por grupo de edad.

La fecundidad es una medida de la capacidad de reproducción de los individuos. En biología pesquera se acepta el concepto de que existe una fecundidad total o absoluta, a nivel de los individuos, que está definida como el número de huevos (ovocitos maduros) que están contenidos en los ovarios y por otro lado la fecundidad relativa, 
que se refiere al número de huevos por unidad de peso o de longitud (5).

La fecundidad se puede estimar a través de varios métodos. En el caso de tratarse de peces pequeños con ovocitos grandes y poco numerosos, pueden efectuarse conteos totales. En el caso de peces que posean ovarios grandes y gran número de ovocitos pequeños se toman submuestras del ovario, y la fecundidad se estima mediante técnicas gravimétricas o volumétricas. Estas se basan en pesar el ovario maduro, o medir su volumen y contar la cantidad de ovocitos presentes en dicha submuestra de cierto volumen o peso. A su vez estos métodos pueden ser húmedos o secos dependiendo de como se manipulen los ovocitos (6).

Para estimar la fecundidad, y por ende para realizar el conteo de los ovocitos maduros se pueden distinguir tres métodos principales que son dados por Bagenal y Braum (6), tomándose solo una submuestra del ovario: a) por volumen; b) por área o c) por secado. Otro método que constituye una técnica húmeda volumétrica, es el denominado de empaquetamiento esférico dado por Guerrero (7) y por Freyre et al (8). En el mismo se homogeniza la muestra, se mide el volumen total ocupado por los ovocitos en una probeta y se realiza el conteo de la misma en una submuestra.

El presente trabajo tuvo como objetivo estudiar el ciclo sexual y la fecundidad del pejerrey patagónico, especie de alto valor comercial y deportivo, en el embalse Ezequiel Ramos Mexía (Neuquén, Río Negro; Argentina)

\section{MATERI ALES Y MÉTODOS}

Época de desove. Para establecer el estado de maduración y el comportamiento de los ovarios y testículos, se realizó el seguimiento de los mismos a través del tiempo. Con este fin, se estudiaron 9307 ejemplares capturados en el embalse Ezequiel Ramos Mexía. Este se encuentra ubicado entre los $39^{\circ} 15^{\prime}$ y $39^{\circ} 40^{\prime} \mathrm{S}$ y los $68^{\circ} 40^{\prime}$ y $68^{\circ} 20^{\prime} \mathrm{O}$ (provincias del Neuquén y Río Negro, Argentina) y su función principal es la de generar energía hidroeléctrica. Las capturas fueron realizadas con baterías de redes agalleras de 42, 50, 60, 70, 78 y $104 \mathrm{~mm}$ de longitud de malla estirada. Las enmalladoras fueron colocadas generalmente a flote, perpendicular a la costa y con la menor malla hacia la misma. El tiempo de calado fue de alrededor de 15 horas. A cada uno de los ejemplares se les recolectaron los siguientes datos: sexo, estado de madurez sexual, longitud total, estándar, peso total, fecha de captura, arte de pesca utilizado y lugar donde se realizó el muestreo.

En la determinación del estado de madurez sexual se siguió el criterio de las observaciones macroscópicas, las mismas fueron realizadas en el laboratorio húmedo. (Tabla 1). Para estudiar los distintos estados de madurez de las gónadas, se adoptó la escala diseñada por Calvo y Dadone (9), la cual describe 7 estados de madurez sexual para el pejerrey bonaerense.

Fecundidad absoluta. Con el fin de estudiar la fecundidad se recolectaron muestras de gónadas (ovarios) de 43 pejerreyes patagónicos. Las muestras fueron depositadas en el laboratorio de Biología de Poblaciones del Instituto de Limnología “Dr. Raúl Ringuelet” (I LPla).

Una vez retiradas las redes del agua se procedió a desenmallar la captura, colocándo los ejemplares en cajones previamente rotulados con el número correspondiente al tamaño de red. Luego se llevaron los mismos al laboratorio húmedo donde se procesaron tomándose sus datos merísticos, sexo y estado gonadal. En el caso de hembras maduras sexualmente se procedió a retirar los ovarios, los cuales se colocaron en formaldehído al $10 \%$, en frascos rotulados dentro de bolsas.

Los ovarios de ejemplares cuyos estados de madurez sexual eran IV o V, fueron utilizados para estimar la fecundidad, descrito por Calvo y Dadone (9), Calvo y Morriconi (10) y en López et al (11) (Tabla 1). 
Los aterinomorfos presentan la característica de poseer la membrana externa de los óvulos recubiertos por filamentos. Esto produce el aglutinamiento de aquellos, impidiendo la homogenización del ovario, causando un error al medir el volumen que ocupan los ovocitos maduros con que luego se trabajará. Además se conoce que existe una distribución espacial de los ovocitos dentro del ovario, asociado a su madurez (12). Para subsanar este inconveniente, se optó por desarrollar una técnica para la separación de los filamentos. Esta consistió en realizar un homogenato con los ovarios, utilizando para este propósito una procesadora cuyas cuchillas se encontraban desafiladas, para no provocar daños a los ovocitos. Una vez finalizado este proceso, se pasa al producto por una serie de tamices de diferentes poros: en primer lugar uno de $5 \mathrm{~mm}$ de lado, luego de $2 \mathrm{~mm}$ y por último de $1 \mathrm{~mm}$. En el primero de los tamices se extrajo manualmente el

Tabla 1. Características macroscópicas en los distintos estados y grados de madurez de las gónadas.

\begin{tabular}{|c|c|}
\hline $\begin{array}{l}\text { ESTADO Y GRADO } \\
\text { DE MADUREZ }\end{array}$ & HEMBRAS \\
\hline Juvenil I & $\begin{array}{l}\text { Testículo muy delgado acintado, Ovarios delgados y cilíndricos, de color } \\
\text { transparente o ligeramente rosado tenue o transparente resaltando la } \\
\text { blancuzco en las partes más arteria genital y sus ramificaciones. } \\
\text { gruesas sanguínea visible. } \\
\end{array}$ \\
\hline En Preparación II & $\begin{array}{l}\text { Color blanquecino rosado. Opacos, Aumentan largo y espesor Son cilíndricos, } \\
\text { sección triangular. } \\
\text { levemente festoneados, arteria Irrigación transversal visible. Ovocitos } \\
\text { genital bien visible. }\end{array}$ \\
\hline En Maduración III & $\begin{array}{ll}\text { Blanco grisáceo, son más opacos, Voluminosos, opaco amarillento. Las } \\
\text { bordes siempre irregulares. Aún se ramificaciones de los vasos son menos } \\
\begin{array}{ll}\text { destaca la red sanguínea } & \text { videntes Ovocitos distinguibles a simple } \\
& \text { vista }\end{array}\end{array}$ \\
\hline Prefreza IV & $\begin{array}{l}\text { Blanco grisáceo o blanco lechoso. } \\
\text { Son voluminosos. Los bordes son Son muy voluminosos, turgentes, ocupan } \\
\text { mas redondeados por el mayor gran parte de la cavidad abdominal, } \\
\text { grosor de las paredes, formándose sección cilindrica. Amarillo verdoso } \\
\text { lobulaciones, separadas. Disminuye Ovocitos grandes translúcidos, con gotitas } \\
\text { la ramificación sanguínea visible. de aceite concentradas en un polo. } \\
\text { Por fuerte presión se puede lograr } \\
\text { emisión de esperma. }\end{array}$ \\
\hline Freza V & $\begin{array}{ll} & \begin{array}{l}\text { Voluminosos y turgentes. A leve presión } \\
\text { expulsan ovocitos maduros. Color } \\
\text { verdoso translúcido en la parte ventral }\end{array} \\
\text { Llenan casi toda la cavidad ocupada por ovocitos maduros. En parte } \\
\text { abdominal. Color blanco lechoso. dorsal a ambos lados de la arteria hay } \\
\text { Los bordes son festoneados. El acumulación de ovocitos inmaduros de } \\
\text { esperma fluye a la menor presión color amarillento. Los ovocitos maduros } \\
\text { son translúcidos, verdosos con gotitas de } \\
\text { aceite más grandes y reunidas en un solo } \\
\text { grupo. }\end{array}$ \\
\hline Post Freza VI & 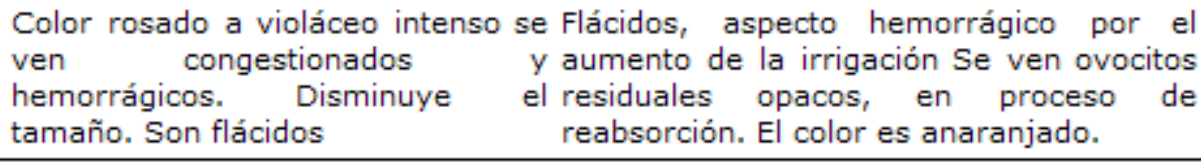 \\
\hline Regresión (II) VII & $\begin{array}{l}\text { Marcada regresión de los testículos, } \\
\text { pequeños, triangulares, bordes } \\
\text { ligeramente festoneados, Cilíndricos con pared ovárica casi oscura, } \\
\text { blanquecinos, la arteria y vena casi negra. Irrigación escasa. Tamaño } \\
\text { testicular siguen un curso irregular reducido. Después Se acerca al estado II. } \\
\text { pasando de una cara a la otra. } \\
\text { Después se acerca al estado II. }\end{array}$ \\
\hline
\end{tabular}


estroma ovárico, en el segundo quedaban retenidos los ovocitos maduros y en el tercero quedaban los ovocitos más pequeños que no habían madurado. Posteriormente se tomaban los ovocitos maduros y se los colocaba en una probeta graduada, se dejaba decantar hasta conseguir el máximo empaquetamiento y luego se leyó el volumen que ocupaban los ovocitos.

En el paso siguiente, se tomó una submuestra al azar del homogenato purificado y se midió el diámetro de unos 45 ovocitos, con la ayuda de un proyector óptico de perfiles con un aumento de 20X. El promedio de este diámetro se consideró el tamaño de un ovocito estándar para esa muestra.

Para calcular la fecundidad se utilizaron las fórmulas propuestas por Guerrero (7) y Freyre et al (8).

$$
F=N . C
$$

donde:

F: es el número total de ovocitos correspondientes a los dos ovarios.

$\mathrm{N}$ : es él número de huevos en la bicapa.

$\mathrm{C}$ : es él número de las bicapas.

$$
N=1,80751836 \times\left(\frac{r_{p}}{r_{\mathrm{m}}}\right)^{2}-3,40964912 \times\left(\frac{r_{p}}{r_{\mathrm{mo}}}\right)
$$$$
C=\frac{\frac{V l(m l)}{\pi \times r_{g}(m m)}-r_{\mathrm{rm}}(\mathrm{mm})}{2 \times \sqrt{3-\tan 30^{\circ}}}+\frac{1}{2}=\frac{\frac{V l(m l)}{\pi \times r_{g}(\mathrm{~mm})}-r_{\mathrm{rm}}(\mathrm{mm})}{1,55648634}+\frac{1}{2}
$$

siendo

$r_{p:}$ radio interno de la probeta

$r_{\text {mo: }}$ radio medio del ovocito

Vol: volumen en $\mathrm{ml}$ desplazado por los ovocitos

El modelo de ajuste entre la fecundidad ( $F)$ $y$ el peso total (W) fue lineal (6).

$$
F=a+b \times W
$$

donde:

a y b: son constantes
Este modelo fue ajustado aplicando el modelo de regresión funcional propuesto por Ricker (13).

En el caso del modelo aplicado entre (F) y la longitud total $\left(L_{t}\right)$, se parte del modelo anterior y se llega a:

$$
\begin{aligned}
& W=A \times L^{B} \\
& F=a+b \times A \times L^{B} \\
& C=b \times A \\
& F=a+C \times L_{t}{ }^{B}
\end{aligned}
$$

donde:

C, a y B son constantes

En este caso se utilizó el modelo de ajuste no lineal de mínimos cuadrados (método iterativo de Newton).

Asimismo se halló la relación existente entre la fecundidad y el peso del ovario

$$
F=a+b \cdot P_{g}
$$

donde:

a y b son constantes

Fecundidad relativa. Se calcularon las fecundidades relativas $\left(F_{R}\right)$ como el cociente entre la fecundidad observada y el peso total (W) en gramos.

$$
F_{R}=\frac{F o b s}{W}
$$

Sé graficó la fecundidad relativa $\left(F_{R}\right)$ vs., la longitud total $\left(L_{t}\right)$ y se buscó la relación que existe entre ambas variables.

Talla de primera madurez sexual. La talla de primera madurez sexual indica a que tamaño las hembras están activas sexualmente por primera vez. Se determinó con ejemplares capturados en los meses en los cuales los individuos se encontraban maduros sexualmente o en vías de estarlo, analizándose un total de 1486 individuos. Se eligieron las hembras por considerarlas mas confiables que los machos para determinar su estado gonadal, considerando 
que los cambios sufridos en los ovarios a lo largo del tiempo son más evidentes que en los testículos.

Se estimó agrupando los datos de todos los años, según intervalos de $L_{t}(10 \mathrm{~mm})$, las hembras que no presentaban signos de maduración (estados I y II) por un lado y por el otro las que presentaron signos de maduración y regresión ovárica (estados III, IV, V, VI y VII). De esta manera se obtuvo el porcentaje de individuos con signos de maduración para cada intervalo de talla. Estos datos fueron ajustados a una curva logística (14).

$$
M=\frac{100}{1+m \cdot e^{-a \cdot L_{t}}}
$$

Para el ajuste del modelo se utilizó un método de regresión no lineal por mínimos cuadrados (método iterativo de Newton).

La talla o $L_{t}$ que correspondió al $50 \%$ (porcentaje de hembras maduras) en la curva logística, se consideró como la talla de primera madurez sexual $(12,15)$.

Proporción de sexos. Se calculó el porcentaje de hembras respecto al total de los ejemplares capturados, este procedimiento se llevó a cabo para cada intervalo de talla y con la totalidad de los individuos capturados en este estudio.

\section{RESULTADOS}

Se describieron las características propias de los ovarios y testículos, para cada estado y grado de madurez sexual diferenciados (Tabla 1 ).

Época de desove. Se graficaron los porcentajes correspondientes a los distintos estados gonadales, tanto para hembras como para machos, en cada uno de los muestreos realizados (Figuras 1 y 2 ).

Se determinó así la época y duración del desove, que es el período donde se encuentra el mayor porcentaje de individuos en estado IV (prefreza) o V (freza).

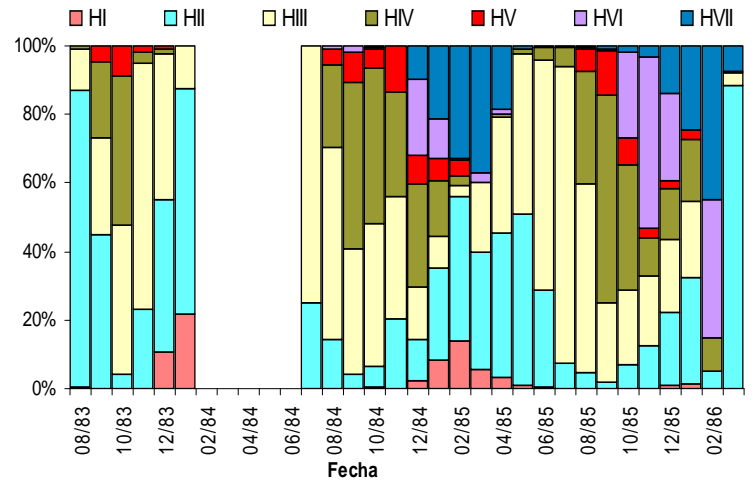

Figura 1. Porcentaje de los distintos estados de las gónadas en hembras.

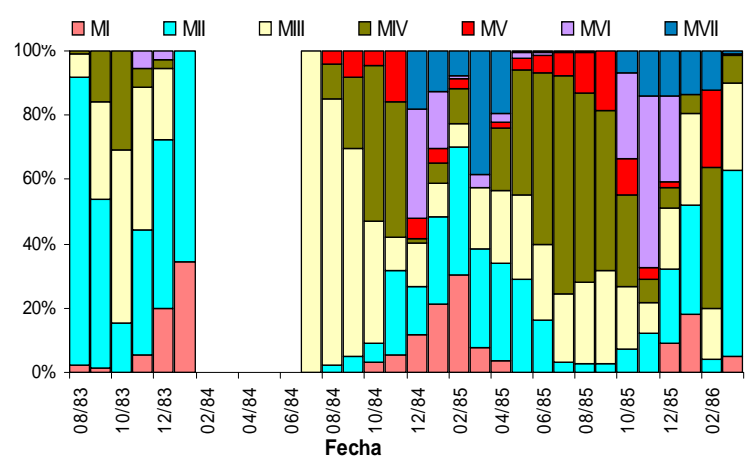

Figura 2. Porcentaje de los distintos estados de las gónadas en machos.

Se evidenció que la proporción de individuos correspondientes al estado II de madurez sexual, fueron bajos entre los meses septiembre-noviembre (Figuras 1 y 2 ). El porcentaje del estado III fue alto entre los meses de mayo y julio. Para el estado IV se observó un alto porcentaje entre septiembre, octubre y noviembre. En cuanto al estadio VII (que corresponde al post-desove), se incrementó entre diciembre y marzo.

Por lo tanto, es notorio que el pejerrey patagónico para el embalse Ezequiel Ramos Mexía posee una sola época de reproducción, entre los meses de septiembre a diciembre, abarcando aproximadamente cuatro meses.

Fecundidad absoluta y relativa. Para los 43 individuos analizados las tallas totales oscilaron entre 296 y $430 \mathrm{~mm}$ y las tallas estándares entre 250 y 365 $\mathrm{mm}$. El diámetro promedio de los ovocitos maduros fue de $1.90 \mathrm{~mm}$. 
La relación fecundidad (F) vs. longitud total $\left(L_{t}\right)$ se observa en la figura 3.

$F=-76302,237+5733,577 \times L_{t} 0,465$

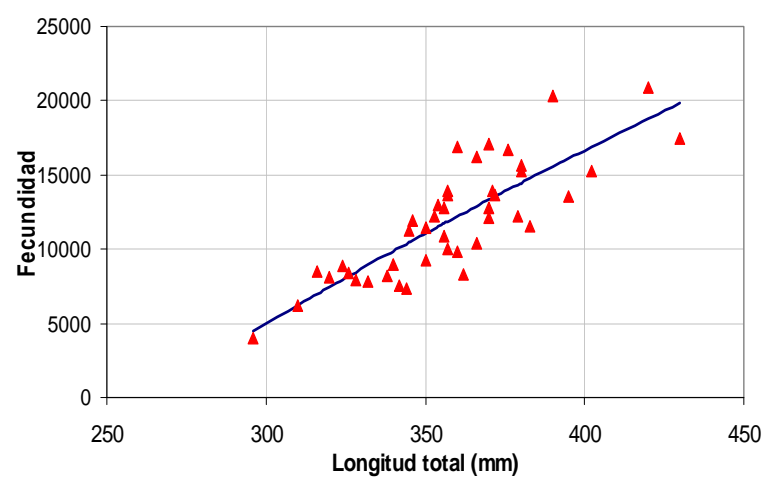

Figura 3. Relación fecundidad (F) vs. longitud total $\left(\mathrm{L}_{\mathrm{t}}\right)$.

$\mathrm{R}^{2}=0,683 ; \mathrm{N}=43$

Para la relación fecundidad (F) vs. peso (W), se probaron dos modelos de ajuste, lineal y potencial, siendo este último el que logró un mejor ajuste de los datos (Figura 4).

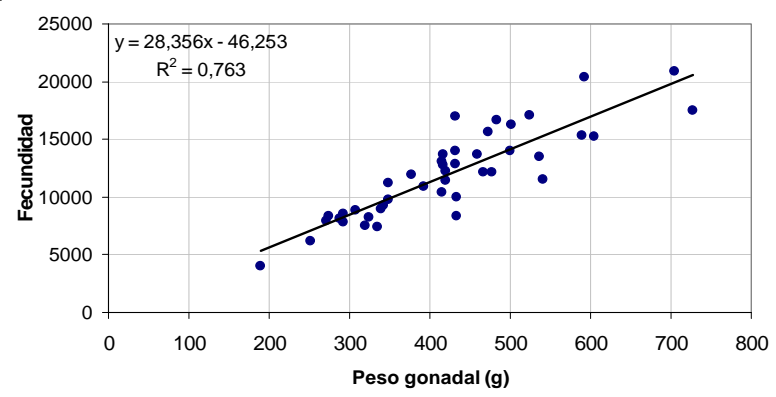

Figura 4. Relación fecundidad (F) vs. peso (W).

Para la relación fecundidad (F) vs., peso del ovario $(\mathrm{Pg})$, se ajustaron los datos a una relación lineal (Figura 5).

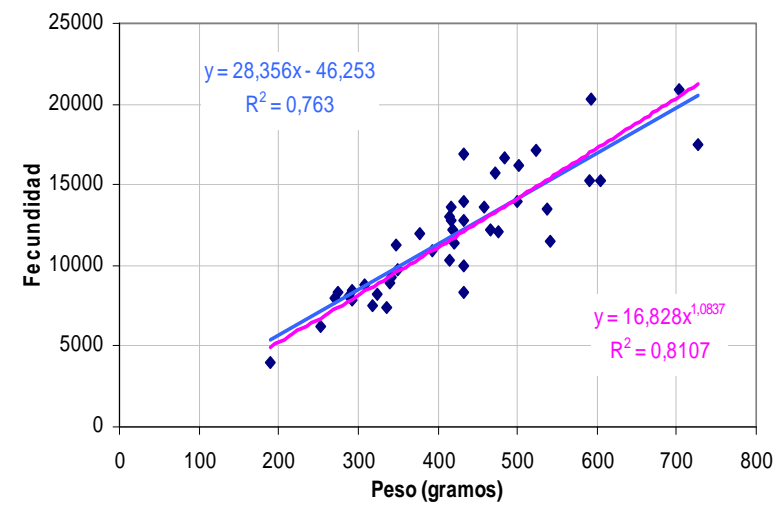

Figura 5. Relación fecundidad (F) vs. peso del ovario $(\mathrm{Pg})$.
Talla de primera madurez sexual. LoS resultados del ajuste al modelo logistico fueron los siguientes:

$$
M=\frac{100}{1+198.77 \times e^{-0.021656 \times L_{t}}}
$$

donde:

$M=\%$ de hembras sexualmente maduras;

$\mathrm{N}=1486 ; r=0,698$ (Figura 6).

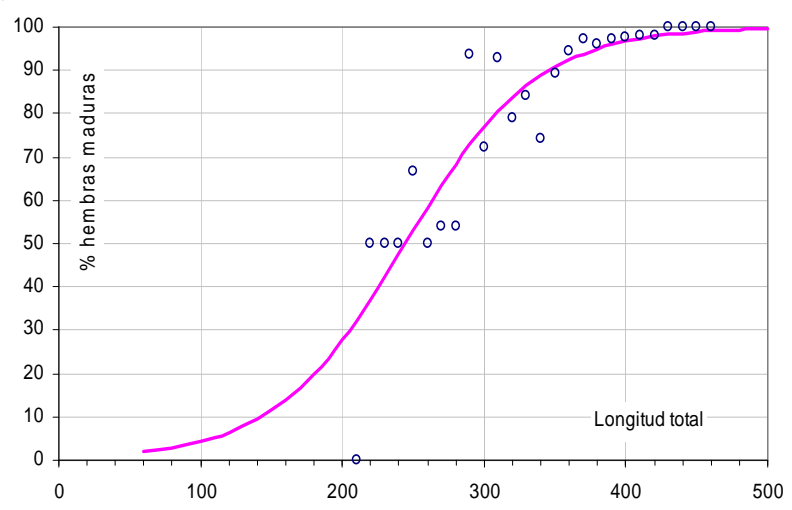

Figura 6. Edad o talla de primera madurez es la talla que alcanza el $50 \%$ de hembras maduras.

Proporción de sexos. En el análisis del porcentaje de hembras a lo largo de los cuatro años en que se tomaron muestras, en función de los intervalos de tallas totales, se observó que en las tallas intermedias entre 220 y $370 \mathrm{~mm}$ de $L_{t}$, la proporción de sexos fue de alrededor del $50 \%$, pero a tallas inferiores osciló entre 0 y $65 \%$ y para tallas mayores fue entre 60 y $100 \%$. Estos resultados evidencian que las tallas menores son poco susceptibles de ser capturadas por las artes empleadas, debido a la alta selectividad de las redes. En la captura de individuos de tallas mayores, la proporción de hembras es más elevada que los machos probablemente debido a la baja frecuencia de captura de estos individuos o tal vez porque que las hembras sean mas fácilmente capturables, debido a un comportamiento más gregario en las tallas mayores (Figura 7).

Asimismo, la proporción de hembras activas reproductivamente respecto al de hembras totales, aumentó a medida que adquirieron una talla mayor (Figura 7). 


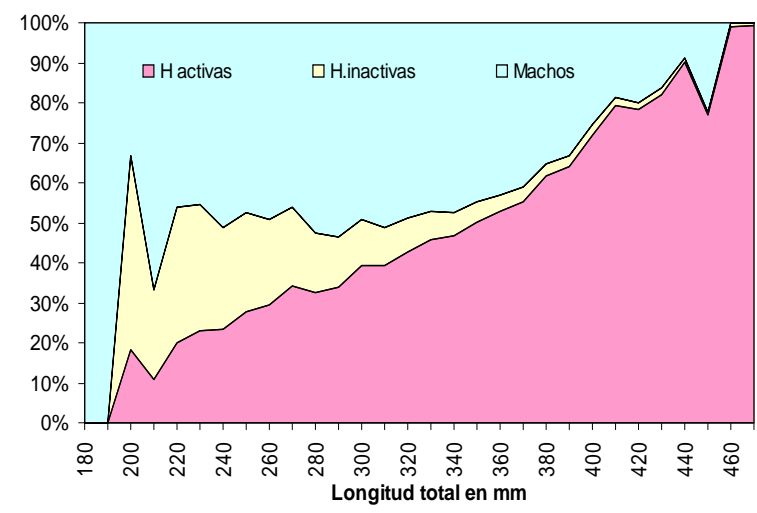

Figura 7. Proporción de hembras activas e inactivas y machos en porcentaje.

\section{DISCUSIÓN}

De acuerdo con las observaciones macroscópicas realizadas en las gónadas del pejerrey patagónico del embalse Ezequiel Ramos Mexía, se describieron siete estados de madurez sexual, a diferencia de lo propuesto por Ruiz (4), quien estableció seis estados de madurez sexual y consideró que unificando el estadio IV y V, se evitan errores de interpretación en el campo.

Se observó que el ciclo ovárico es altamente estacionalizado y que probablemente responda a variables ambientales, tales como temperatura y fotoperíodo, que en esta región son marcadas por tratarse de un clima templado frío. La época de preparación y maduración se evidenció entre finales del verano, durante el otoño y el invierno. En tanto la temporada de desove ocurrió entre los meses de septiembre y diciembre (primavera y principios del verano), donde el porcentaje de individuos maduros fue mayor (estados IV y V). Estas observaciones son similares a las encontradas por Ruiz (4) en el embalse Florentino Ameghino, donde estableció que el período de preparación es en el otoño, el período de maduración con puestas parciales es en el invierno tardío y primavera y el período donde encuentram las gónadas en reversión es en el verano. Estos resultados se corresponden con los encontrados por Viozzi (1) en el Lago Pellegrini (Neuquén), donde el valor máximo del índice gonadosomático (IGS) se da entre los meses de agosto a diciembre y por las de Bello et al (3) en el lago Moreno (Río Negro). Similar, a lo realizado por López et al (11), en el embalse Ezequiel Ramos Mexía, que obtuvo el valor máximo del índice gonadosomático (IGS), entre los meses de septiembre y octubre.

Grosman et al (16), determinaron que el periodo reproductivo del pejerrey patagónico en la laguna Terraplén (Provincia de Chubut), es corto y abarca los meses de octubre y noviembre. Del Valle y Núñez (17) indicaron que la época de reproducción de esta especie ocurre entre los meses de agosto y enero. Asimismo, en el pejerrey bonaerense las épocas de desove ocurren en primavera y en otoño (10). En este aspecto ambas especies de pejerrey coincidirían sólo con la época de desove de primavera.

La talla de primera madurez sexual de los individuos analizados para este trabajo fue de $244.37 \mathrm{~mm}$ de longitud total, que corresponde al $50 \%$ y represento el tamaño medio para el cual las hembras de la población han entrado en actividad sexual. Las observaciones realizadas en el pejerrey patagónico del embalse F. Ameghino indicaron que la talla media de primera madurez sexual fue de $224.89 \mathrm{~mm}$ (4); mientras que la talla de primera madurez sexual, en el caso de los peces capturados en el lago Moreno fue de $230 \mathrm{~mm}$ (3), ambos valores un poco menores que las encontradas en el presente estudio.

No se observó un patrón definido entre las variables, fecundidad relativa y la longitud total. En cambio si se observaron altos valores de coeficientes de determinación, al evaluar la asociación entre la fecundidad y el peso total $\left(R^{2}=0.8107\right)$, la fecundidad y el peso gonadal $\left(R^{2}=0.763\right)$, y entre la fecundidad y la longitud total $\left(R^{2}=0.683\right)$.

Respecto a la proporción de sexos se puede interpretar que la mayor parte de la población posee un $50 \%$ de individuos hembras y un $50 \%$ de individuos machos. Sin embargo, a tallas menores de 220 $\mathrm{mm}$ y mayores de $360 \mathrm{~mm}$, fue difícil determinar la proporción de sexos, debido a la escasa capturabilidad de individuos correspondientes a estas tallas. Asimismo 
se observó que la actividad sexual de las hembras comienza a los $190 \mathrm{~mm}$ de longitud total, para luego incrementarse cuando adquieren una mayor talla, llegando a casi el $100 \%$, a los $400 \mathrm{~mm}$ de $\mathrm{L}_{\mathrm{t}}$.

En cuanto a las características reproductivas el pejerrey patagónico se encuentra dentro de los denominados desovantes parciales. Como un comentario adicional se puede decir, que la fecundidad absoluta varío entre 3976 huevos/hembra $\left(L_{t}=296 \mathrm{~mm}\right.$ y W $\left.=190 \mathrm{~g}\right)$ a 20879 huevos/ hembra $\left(L_{t}=420 \mathrm{~mm}\right.$ y $\left.W=704 \mathrm{~g}\right)$. Estos valores son mayores a los obtenidos por Bello et al (3) al analizar ejemplares de esta misma especie provenientes del Lago Moreno, ya que fluctuaron entre 1376 a 4788 huevos/hembra, aunque los peces estaban comprendidos entre los 335 y 400 $\mathrm{mm}$ de longitud total.

Para el pejerrey bonaerense las cantidades promedio de ovas producidas son: para ejemplares de $250 \mathrm{~mm}$ de $\mathrm{L}_{\text {est }} 8000$ y para ejemplares de $300 \mathrm{~mm}$ de $\mathrm{L}_{\text {est }} 14.000$, (18). En conclusion, no se observó una relación significativa entre la fecundidad relativa y la longitud total de los ejemplares. La proporción de sexos resultó equilibrada en las clases de longitud intermedias, pero mostró una preponderancia de hembras en las mayores. El ciclo ovárico es altamente estacional y coincide con el de poblaciones de la especie en otros ambientes patagónicos. La fecundidad, en cambio, resultó más elevada que la observada en el lago Moreno, situado en la región cordillerana.

\section{Agradecimientos}

A la empresa Hidronor por haber financiado este trabajo, (convenio Hidronor-Museo Argentino de Ciencias Naturales "Bernardino Rivadavia") y al Lic. Roberto Sauval por haber facilitado parte de los materiales para esta investigación.

\section{REFERENCIAS}

1. Viozzi G. Infestación parasitaria de Patagonina hatcheri (Pisces Atherinidae) en el lago Pellegrini, Río Negro. [Tesis de Licenciatura]. Río Negro: CRUB, Univ. Nac. del Comahue; 1993.

2. Bello MT, Alonso MF, Macchi PJ . Estado del pejerrey patagónico introducido en la laguna Ñe Luán (Río Negro). En: I Congreso y III Reunión Argentina de Limnología. Tucumán Tankay 1. 1994; 219-221.

3. Bello MT, García AA, Alonso MF. Aspectos de la biología reproductiva del pejerrey patagónico Odontesthes hatcheri (Eig.). En: III. Jorn. Patag. Medio Ambiente, Esquel. 1996; p 50.

4. Ruiz AE. Biología del pejerrey patagónico, Odontesthes hatcheri (Eigenmann, 1909) Dyer, 1993, en el embalse Florentino Ameghino, Chubut, Argentina. [Tesis Doctoral]. Córdoba: Universidad Nacional de Córdoba, Facultad de Ciencias Exactas, Físicas y Naturales; 2002.

5. Rabinovich JE. Introducción a la ecología de poblaciones animales. México: CECSA; 1980.

6. Bagenal TB, Braum E. Eggs and early life history. En: T. B. Bagenal (ed.). Methods for assesment of fish production in freshwaters. IBP Handbook №3. Blackwell Scientific Publications Ltd; 1978.

7. Guerrero CA. Demografía y dinámica de la población de la perca de boca grande Percichthys colhuapiensis del embalse Ezequiel Ramos Mexía. [Tesis doctoral]. Buenos Aires: Facultad de Ciencias Exactas y Naturales (U.B.A); 1989. 
8. Freyre LR, Guerrero CA, Maroñas ME. Propuesta de una corrección para la estimación del número de huevos por el método volumétrico. En: Jornadas de Biología del pejerrey IIB-INTECH, Chascomús; 2004.

9. Calvo J, Dadone L. Fenómenos reproductivos en el pejerrey (Basilichthys bonariensis) I. Escala de Tabla de Madurez. Rev. Museo de la Plata, Sección Zoología. 1972; XI: 153-163.

10. Calvo J, Morriconi ER. Fenómenos reproductivos en el pejerrey (Basilicthys bonariensis) III. Estudio de la fecundidad época y numero de desoves. Anales de la Sociedad Cientfica Argentina 1972; (1-2) 75-84.

11. López RB, Torno A, Guerrero C, López G, Ferriz R. Necton-peces. Plan de estudios ecológicos de la cuenca del río Negro Museo Argentino de Ciencias Naturales "Bernardino Rivadavia" Tomo III. [I nforme final]. Buenos Aires: HIDRONOR S. A.; 1981.

12. Freyre LR, I waszkiw J M. I mportancia de la distribución especial de ovocitos en ovarios de peces, para determinaciones de fecundidad. Limnobios 1982; 2 (5): 305-310.
13. Ricker WE. Computation and interpretation of biological statistics of fish populations. J Fish Res Board Can 1975; 191: 1- 382.

14. Berkson J.Application of the logistic function to biossay. J Am Stat Assoc 1944; 39: 357-365.

15. I waszkiw JM, Freyre LR, Sendra ED. Estudio de la maduración, época de desove y fecundidad del dientudo, Oligosarcus jenynsii (Pisces Characidae) del embalse Río III, Córdoba, Argentina. Limnobios, 1983; 2(7): 518-525.

16. Grosman MF, Rudzik G. Análisis de la dieta del "Pejerrey patagónico" Patagonina hatcheri Eigenmann, 1909, Atherinidae, de la laguna Terraplén, Chubut, Argentina. Biota 1990, 6: 71-88.

17. Del Valle AE, Nuñez P. Los peces de la provincia del Neuquen. [Informe Técnico 1]. Buenos Aires: Hemisferio Sur S.A. 1990.

18. Grosman F, (Editor). El pejerrey, ecología, cultivo, pesca y explotación. Azul. Astianax 1995. 